IDDF2020-ABS-0195 EFFICACY AND SAFETY OF LOW-DOSE THALIDOMIDE COMBINED WITH MESALAZINE IN THE TREATMENT OF REFRACTORY ULCERATIVE COLITIS IN ADULTS

${ }^{1}$ Junrong Chen*, ${ }^{2}$ Lei Mai, ${ }^{1}$ Jiachen Sun, ${ }^{1}$ Xiang Peng, ${ }^{1}$ Min Zhang, 'Min Zhi. ${ }^{1}$ The Sixth Affiliated, Sun Yat-sen University, China; ${ }^{2}$ The Fifth Affiliated, Sun Yat-sen University, China

\subsection{6/gutjpl-2020-IDDF.117}

Background To evaluate the efficacy and safety of low-dose thalidomide combined with mesalazine in the treatment of refractory adult ulcerative colitis(UC).

Methods The refractory adult UC patients treated with lowdose thalidomide combined mesalazine from Jan. 2018 to May. 2020 were included. Their clinical records such as the clinical characteristics, course of treatment, efficacy and adverse reactions were reviewed.

Results Among the 14 patients with refractory UC in adults, 9 males, 5 females, 14 total colon involvement, 14 chronic relapse type, the average duration were 7.47 years, and the average age was 45 -years old, while all patients had previously received adequate amounts of hormone-induced remission. 14 active UC patients were treated with low dose thalidomide (25-50 mg/d p.o) combined with mesalazine on the premise that sufficient mesalazine $(\geq 4 \mathrm{~g} / \mathrm{d}$ p.o) was ineffective for 2 weeks. After treatment, the median onset time was 3.5 weeks and the clinical remission rates within 8 weeks and mucosal healing rate by endoscopy was $78.6 \%(11 / 14)$ and $62.5 \%(5 / 8)$ respectively. Among the 3 patients with ineffective treatment, 1 patient was handled by infliximab injection and 2 by surgery. 3 patients had ADRs, all of which were nerve damage, and were taken off medication, and 1 patient had disease recurrence and was reclassified to infliximab treatment.

Conclusions Low-dose thalidomide combined with mesalazine is effective in the treatment of refractory adult UC, which can be used to induce remission and promote mucosal healing, with few and can be tolerated by most patients. However, in clinical application, it is necessary to select the right group and closely monitor ADRs during treatment.

\section{IDDF2020-ABS-0196 EFFECTIVENESS OF VONOPRAZAN AND LOW DOSE AMOXICILLIN DUAL THERAPY AS FIRST LINE AGENTS AGAINST HELICOBACTER PYLORI: A META-ANALYSIS}

Matthew Aguila Lee*, Ma Regina Dimaculangan. Department of Medicine, St. Luke's Medical Center Global City, Philippines

\subsection{6/gutjnl-2020-IDDF.118}

Background Helicobacter pylori infection is one of the common chronic bacterial infections in humans. The standard treatment includes a triple therapy regimen with at least two different antibiotics and one proton pump inhibitor (PPI). However, dual therapy composed of a PPI and Amoxicillin could be used as an alternative for $H$. pylori treatment. Because it is a single antibiotic therapy, there would be less chance for $\mathrm{H}$. pylori antibiotic resistance. This study aims to determine the efficacy and safety of the Vonoprazan-based dual therapy compared to the triple therapy for Helicobacter pylori treatment.

Methods We performed a systematic search in PubMed and the Cochrane Library databases for relevant studies up to
August 2020. Studies were included if they compared the efficacy of $\mathrm{H}$. pylori eradication of dual therapy with Vanoprazan and Amoxicillin and triple therapy with Vanoprazan, Amoxicillin, and Clarithromycin

Two randomized controlled trials comparing the efficacy of the dual and triple Vonoprazan-based therapies published from 2018 were reviewed in this meta-analysis. Both studies compared the use of dual therapy with Vonoprazan and Amoxicillin with the triple therapy composed of Vonoprazan, Amoxicillin, Clarithromycin in determining the eradication rate of H.pylori and other significant adverse effects. Studies were analyzed in the group to which they were originally randomized using the intention to treat analysis.

Results Two studies with 517 patients were evaluated in this meta-analysis. The $\mathrm{H}$. pylori eradication rate of dual therapy was non-inferior than that of triple therapy as first-line regimens (intention-to-treat analysis: pooled eradication rates, 88\% vs 90\%; odds ratio [OR], 0.98; 95\% confidence interval (CI): [0.53-1.64]; $\mathrm{P}<0.05)$. The pooled evidence of this meta-analysis showed that eradication of $\mathrm{H}$. Pylori infection using dual therapy with Vanoprazan and Amoxicillin compared to triple therapy with Vanoprazan, Amoxicillin and Clarithromycin did not have a significant difference.

Conclusions The dual therapy with Vonoprazan and low-dose Amoxicillin provided acceptable $H$. pylori eradication rates and a similar effect to Vonoprazan-based triple therapy.

\section{IDDF2020-ABS-0202 META-ANALYSIS ON THE EFFECT OF PROBIOTICS ON NEURODEGENERATIVE DISORDERS IN HUMANS CLINICAL TRIALS}

${ }^{1}$ Henry Yue Hong Meng*, ${ }^{2}$ Chi Hang Christopher Mak, ${ }^{3}$ Joyce Wing Yan Mak, ${ }^{4}$ Owen Ho Ko, ${ }^{5}$ Zuo Tao, ${ }^{3}$ Francis Ka Leung Chan. ${ }^{1}$ Faculty of Medicine, The Chinese University of Hong Kong, Hong Kong; ${ }^{2}$ School of Clinical Medicine, University of Cambridge, UK; ${ }^{3}$ Center for Gut Microbiota Research, Department of Medicine and Therapeutics, Institute of Digestive Disease, The Chinese University of Hong Kong, Hong Kong; ${ }^{4}$ Division of Neurology, Department of Medicine and Therapeutics, Faculty of Medicine, The Chinese University of Hong Kong, Hong Kong; ${ }^{5}$ Department of Medicine and Therapeutics, Institute of Digestive Disease, LKS Institute of Health Science, The Chinese University of Hong Kong, Hong Kong

\subsection{6/gutjnl-2020-IDDF.119}

Background Probiotics have been shown to improve neurocognitive behaviour in animal models via the gut-brain-axis. This meta-analysis aims to evaluate the evidence in human studies on the potential of probiotics as a treatment for age-related neurodegeneration such as Mild Cognitive Impairment (MCI) and Alzheimer's Disease (AD).

Methods A PRISMA meta-analysis was conducted by screening through MEDLINE, Embase, Scopus, Web of Science and Cochrane library for human studies using equivalent combinations of 'probiotics', 'age-related neurodegeneration', 'MCI' and 'AD'. Studies with Mini-mental State Examination (MMSE), a test for cognitive function with comparable quantitative outcome were meta-analysed using RStudio. The analysis for overall effect $(95 \% \mathrm{CI})$ and heterogeneity $\left(\mathrm{I}^{2}\right)$ was performed by Forest Plots and subgroup analysis. We further assessed whether the results varied with age,\%female, BMI, dosage and sample size with weighted meta-regression. Probiotic amelioration of neurodegeneration was also evaluated through biomarker analysis.

Results 9 human studies were identified, where 5 studies had quantitative results. Meta-analysis demonstrates that there is a 